\title{
CARACTERIZACIÓN DE PROTEÍNAS Y CONTENIDO MINERAL DE DOS VARIEDADES NATIVAS DE FRIJOL DE MÉXICO ${ }^{1}$
}

\author{
Juan Carlos Raya-Pérez ${ }^{2}$, Glenda Margarita Gutiérrez-Benicioº ${ }^{2}$ Juan Gabriel Ramírez Pimentel ${ }^{2}$, \\ Jorge Covarrubias-Prieto ${ }^{2}$, César Leobardo Aguirre-Mancilla ${ }^{2}$
}

\section{RESUMEN}

Caracterización de proteínas y contenido mineral de dos variedades nativas de frijol de México. El objetivo de este trabajo fue caracterizar a nivel bioquímico la semilla de dos variedades mexicanas de frijol, Bayo Berrendo y Patzcuareño. En el año 2011, en Celaya, México, se extrajeron proteínas de reserva de las dos variedades criollas de frijol (Phaseolus vulgaris), con el fin de obtener sus perfiles electroforéticos. El contenido de proteína soluble en el caso de Bayo Berrendo fue de 16,47\% y 14,53\% para el Patzcuareño. Se detectaron diferencias en el patrón electroforético, sobre todo en la fracción de albúminas, que permitió diferenciar las variedades. La harina presentó alta actividad inhibitoria contra tripsina, principalmente en la fracción de faseolinas, 355 unidades en el Bayo Berrendo y 507 unidades en el Patzcuareño. Se determinó la composición elemental de la harina observándose diferencias en cuanto al contenido mineral. El Bayo Berrendo tiene un contenido de $0,15 \%$ de $\mathrm{Ca}, 5,53 \%$ de $\mathrm{K}$ y $0,60 \%$ de $\mathrm{Mg}$. Los valores respectivos para el Patzcuareño fueron: $0,17 \%, 3,52 \%$ y $0,67 \%$. El Bayo posee 18,8 ppm de Fe y el Patzcuareño 3,63 ppm. Se midieron otras variables relacionadas con su utilización con fines alimenticios, como capacidad de absorción de agua, con valores cercanos al $100 \%$ y tiempos de cocción menores a 110 min. Fenotípicamente las dos variedades se distinguen fácilmente, tanto en tamaño de semilla como en color. El peso de 100 semillas del Bayo Berrendo fue de 14,6 g y del Patzcuareño 32,6 g.

Palabras clave: contenido mineral en frijol, inhibidores de proteasas, patrón electroforético de proteínas, diversidad en proteínas de frijol nativo.

\begin{abstract}
Characterization of proteins and mineral content of two bean landraces from Mexico. The objective of this work was to characterize, at the biochemical level, two Mexican native landraces Bayo Berrendo and Patzcuareño of common bean. Storage protein from seeds was extracted from two common bean (Phaseolus vulgaris) varieties (Bayo Berrendo and Patzcuareño), and their respective electrophoretic patterns were compared. Total soluble protein was $16,47 \%$ for Bayo Berrendo, and 14,53\% for Patzcuareño. Differences were also detected in the albumin fraction. Moreover their flour showed high inhibitory activity against trypsin mainly in the phaseolin fraction, which corresponded to 355 units in the Bayo Berrendo and 507 units in the Patzcuareño variety. The elemental composition of the flour was also determined and showed differences. The Bayo Berrendo seed flour has a composition of $0,15 \% \mathrm{Ca}, 5,53 \% \mathrm{~K}$ and $0,60 \% \mathrm{Mg}$. While values for the Patzcuareño flour were $0,17 \%, 3,52$ and $0,67 \%$ respectively. Interestingly the Bayo variety contained 18,8 ppm iron, while Patzcuareño contains only 3,63 ppm. Other variables related to food usage were analyzed such as water absorption capacity, which showed values near $100 \%$ for both landraces, and cooking time which took less than 110 minutes. Phenotypically, the two landraces are different in both size and color. The weight for 100 seeds of the Bayo Berrendo variety was 14,6 $\mathrm{g}$ and for Patzcuareño 32,6 g.
\end{abstract}

Key words: bean mineral content, protease inhibitors, electrophoretic protein pattern, diversity in native bean proteins.

1 Recibido: 5 de junio, 2013. Aceptado: 18 de marzo, 2014. Este trabajo forma parte del proyecto "Caracterización de frijol criollo nativo de México".

2 Instituto Tecnológico de Roque, División de Estudios de Posgrado e Investigación. km 8 Carretera Celaya-Juventino Rosas, C.P. 38110, Celaya, Gto., México. Tel/Fax 01461 6117757.juancarlos.raya@gmail.com,glemgb@hotmail.com,drjgrp2004@yahoo.com.mx, jor_ covarru-jrg@hotmail.com, cesar.aguirre.m@gmail.com (autor para correspondencia). 


\section{INTRODUCCIÓN}

Una de las principales fuentes de proteína en Latinoamérica y África es el frijol (Phaseolus vulgaris) (Omae et al., 2012). Además, es fuente de vitaminas, rico en ácido fólico, tiamina, minerales y junto con los cereales complementan el balance de los aminoácidos (Onder y Babaoglu, 2001; Serrano y Goñi. 2004), y proveen de calcio, hierro y zinc a los estratos más pobres de la población. El frijol negro aporta el 19,1\% del Fe y el 15,9\% del Zn de los requerimientos diarios en el caso de la población guatemalteca (Serrano y Goñi, 2004). La anemia a causa de la deficiencia de Fe es reconocida como un problema de salud pública, lo mismo que la carencia de $\mathrm{Zn}$, por lo cual se ha buscado enriquecer la dieta en estos elementos a través de distintas estrategias, entre ellas, cultivar variedades ricas en estos elementos (Guzmán-Maldonado et al., 2000; Jiménez-Hernández et al., 2012). En general, el frijol común presenta niveles adecuados, en relación con la ingesta diaria recomendada de magnesio, potasio y cobre y niveles pobres de sodio, boro, fierro, manganeso y zinc (Amirul-Islam et al., 2006).

Las proteínas de almacenamiento representan en el frijol el 50\% de la proteína que contiene el grano (Samour et al., 2007) y de estas, las albúminas representan un $14,8-20,8 \%$, las globulinas $33-45 \%$, las glutelinas $12,8-41,2 \%$ y las prolaminas en general, menos del 1\% (Perazzini et al., 2008). Entre las globulinas, las faseolinas son las más abundantes y presentan variación tanto en su masa molecular como en el número de subunidades que las conforman y su expresión no depende del ambiente (Montoya et al., 2008). El patrón de bandeo electroforético ha sido usado para la identificación y la búsqueda de relaciones filogenéticas entre especies y entre razas cultivadas y silvestres. La determinación de los perfiles de proteínas es utilizada ampliamente en la identificación de genes que se expresan diferencialmente en plantas sometidas a condiciones distintas de forma natural o experimentales (Guerrero-Zúñiga y Rodríguez-Dorantes, 2009).

El mejoramiento de los cultivos se acelera al ampliar la base genética y la información acerca de los recursos disponibles (Tandang-Silvas et al., 2011, Sammour et al., 2007). En lugares como Italia y España se han hecho estudios para colectar, conocer y proteger sus razas, mismas que fueron introducidos desde América hace unos cinco siglos (Casañas et al., 1999, Perazzini et al., 2008) además de los esfuerzos en otros países para mejorar las cualidades de este cultivo, tanto nutricionales como de reducir la presencia de factores antinutricionales o de flatulencia; esta última debido a la presencia de oligosacáridos (Amirul-Islam et al., 2006; Da Silva-Fialho et al., 2006).

En el caso de México, la situación de abandono del campo por parte de los agricultores y la falta de continuidad en sus cultivos, está provocando la pérdida de información sobre las bondades y el manejo de las variedades cultivadas tradicionalmente, lo que puede contribuir a la pérdida de estas (Rubio, 2006). El conocimiento de la diversidad genética en nuestros cultivares permitirá ampliar la base para los programas de mejoramiento, que hasta ahora, por lo menos en algunos casos, muestra poca variabilidad (VidalBarahona et al., 2006; Omae et al., 2012). La calidad del grano es importante para los consumidores, esta incluye características físicas, químicas y culinarias. El peso de la semilla es una propiedad que se relaciona con la dimensión y densidad de semilla de frijol; esta característica, además, define la calidad del grano por el consumidor. Las semillas con testa de color están mejor protegidas contra patógenos que cuando muestran color albino o blanco (Romano et al., 2010). El valor comercial es influenciado por el tamaño y el color del grano, tiempo de cocción, sabor y espesor del caldo (Pérez-Herrera et al., 2002).

Este conocimiento también podría favorecer los esfuerzos por incrementar el consumo per cápita de esta leguminosa, tratando de disminuir el consumo de comida rápida.

El objetivo de este trabajo fue caracterizar a nivel bioquímico la semilla de dos variedades mexicanas de frijol, Bayo Berrendo y Patzcuareño.

\section{MATERIALES Y MÉTODOS}

Las variedades de frijol seleccionadas para este trabajo fueron el frijol Bayo Berrendo (color gris-rosado), la cual se adquirió en el mercado local de Colima (México), y el Patzcuareño (color café-rosa-pinto) en el mercado local de Uruapan (México). Ninguna de las dos variedades aparece en la colección núcleo del Instituto Nacional de Investigaciones Forestales Agrícolas y Pecuarias (INIFAP, México) (Vargas-Vázquez et al., 2006).

El grano se molió finamente y se desgrasó con una mezcla de cloroformo-metanol (2:1) proporción 4:1 $(\mathrm{p} / \mathrm{v})$. La harina desgrasada se utilizó para la extracción 
de proteínas (Syros et al., 2003). El contenido de humedad se determinó pesando 20 granos e incubando a $80^{\circ} \mathrm{C}$ durante 24 horas, hasta obtener peso constante (Da Silva-Fialho et al., 2006). El peso del embrión, cotiledones y testa se realizó usando la metodología de Pérez-Herrera et al. (2002). La determinación de cenizas se hizo por duplicado por incineración de la muestra a una temperatura de $500^{\circ} \mathrm{C}$.

\section{Extracción y fraccionamiento de las proteínas}

Las proteínas de reserva de las semillas, se extrajeron secuencialmente partiendo de harina desgrasada. Para la extracción de la fracción albúmina se pesó $1 \mathrm{~g}$ de harina y se agregaron $10 \mathrm{ml}$ de agua destilada y se sometió a agitación magnética por una hora a temperatura ambiente $\left(25^{\circ} \mathrm{C}\right)$, posteriormente se centrifugó a $14000 \mathrm{rpm}$ durante quince minutos y se recuperó el sobrenadante (fracción albumina). Para la extracción de la fracción globulina, a la harina precipitada de la extracción anterior se le agregó $10 \mathrm{ml}$ de una solución de cloruro de sodio $0,5 \mathrm{M}$ en Tris $50 \mathrm{mM}$ pH 8 y se sometió a agitación magnética por una hora a $25^{\circ} \mathrm{C}$, posteriormente se centrifugó bajo las mismas condiciones de la extracción anterior y se recuperó el sobrenadante (fracción globulina). Para la extracción de la fracción prolamina, la harina precipitada de la extracción anterior se resuspendió en 2-propanol al $55 \%$ y se extrajo bajo las condiciones descritas en las fracciones anteriores. Finalmente, la fracción glutelina se extrajo resuspendiendo la harina precipitada de la extracción anterior en amortiguador de boratos $0,1 \mathrm{M}$ con SDS al 0,5\%, pH 9, bajo las condiciones descritas anteriormente (Syros et al., 2003).

Para la extracción de proteína total, se pesó $0,1 \mathrm{~g}$ de harina desgrasada y se extrajo con $1 \mathrm{ml}$ de solución amortiguadora que contenía: Tris- $\mathrm{HCl} 250 \mathrm{mM} \mathrm{pH}$ 7 , glicerol al $10 \%(\mathrm{v} / \mathrm{v})$, SDS al $1 \%(\mathrm{p} / \mathrm{v})$ y 2 -mercaptoetanol al 4\% (Sammour et al., 2007); la muestra se colocó durante cinco minutos en agua hirviendo, posteriormente se centrifugó a $13000 \mathrm{rpm}$ durante cinco minutos; se recuperó el sobrenadante para ser sometido a electroforesis en gel de poliacrilamida. La extracción de faseolinas se realizó acorde al método reportado por Montoya et al. (2008) usando harina desgrasada; a 0,1 g de la harina se le agregó 1,2 ml de solución que contenía $\mathrm{NaCl} 0,5 \mathrm{M}$ y $\mathrm{HCl} 0,025 \mathrm{M}$, se incubó a temperatura ambiente con agitación magnética durante una hora y se centrifugó a 13000 rpm durante quince minutos. Se tomó el sobrenadante y se le agregaron cinco volúmenes de agua destilada a $4^{\circ} \mathrm{C}$, obteniéndose la precipitación de la fracción de faseolinas. Este precipitado se recuperó por centrifugación a $13000 \mathrm{rpm}$ durante quince minutos para ser sometido a electroforesis en gel de poliacrilamida.

La proteína de las diferentes extracciones se cuantificó por triplicado mediante el método de Bradford (1976), usando curva de calibración con albúmina de suero bovino.

\section{Extracción de carbohidratos solubles}

Se pesaron 0,25 g de harina, se realizó extracción con etanol al $80 \%$ durante $12 \mathrm{~h}$ a temperatura ambiente, posteriormente, se centrifugó a 10000 rpm durante 10 min en microcentrífuga (Eppendorf ${ }^{\mathrm{MR}}$ modelo 5415D) y se recuperó el sobrenadante. Al residuo de harina se le agregó 0,5 $\mathrm{ml}$ de agua destilada, se dejó reposar una hora y se centrifugó (1000 rpm durante 10 min) y se mezclaron los sobrenadantes (Gonçalves et al., 1997; Hall et al., 1999).

\section{Identificación de carbohidratos}

Los carbohidratos presentes en las muestras se identificaron por cromatografía en capa fina, usando como fase móvil una mezcla de cloroformo-metanolácido acético 70:30:15 y se reveló con una solución revelada de anisaldehído (anisaldehído Sigma-aldrich ${ }^{\mathrm{TM}}$ $5 \%, 90 \%$ etanol y $5 \% \mathrm{H}_{2} \mathrm{SO}_{4}$ concentrado v/v); como referencia se usaron estándares de sacarosa, glucosa, dp3, dp4 y dp5 (fructooligasacaridos de bajo grado de polimerización) (Nierth y Jäschke, 2011).

\section{Electroforesis}

Para la separación de proteínas se emplearon geles de poliacriamida al $10 \%$ en condiciones desnaturalizantes, con amortiguador de tricina (Schägger y von Jagow, 1987). Los geles se fijaron con una mezcla de metano-ácido acético-agua (4:1:5) por $30 \mathrm{~min}$, después se tiñeron con azul de Coomassie al $0,1 \%$ disuelto en ácido acético al $10 \%$ por $2 \mathrm{~h}$.

\section{Determinación de actividad inhibitoria de proteasas}

La actividad de inhibición de tripsina fue determinada con la técnica de Erlanger et al. (1961), 
midiendo el incremento en absorbancia a $253 \mathrm{~nm}$ con el programa Kinetics, en espectrofotómetro marca Beckman. Se incubó 30 min a $30^{\circ} \mathrm{C}$ una alícuota (60 $\mu 1)$ por separado de cada fracción proteica con solución de tripsina bovina (10 $\mu \mathrm{l}) 200 \mu \mathrm{g} / \mathrm{ml}$, en 2,8 $\mathrm{ml}$ de amortiguador de Tris- $\mathrm{HCl} 0,15 \mathrm{M} \mathrm{pH} \mathrm{8,1,y}$ posteriormente, se agregó $50 \mu \mathrm{l}$ del sustrato sintético (BAEE- Ester etílico de N-benzoil-l-arginina 0,014M pH 8,1 ), se incubó 3 min a $30^{\circ} \mathrm{C}$ y finalmente se leyó en espectrofotómetro.

Una unidad de enzima es definida como un incremento de 0,01 de absorbencia a $253 \mathrm{~nm}$. La actividad inhibitoria es definida como el número de unidades de enzima inhibidas. Se calcula con la siguiente fórmula:

$$
\mathrm{UI} / \mathrm{ml}=\frac{\text { Abs Enz }-(\text { Abs Enz }+ \text { muestra })}{0,01 \times \text { volumen de muestra }(\mathrm{ml})}
$$

Donde:

Abs Enz = absorbencia enzima (control)

Abs Enz + muestra = absorbencia enzima + fuente de inhibidores de proteasas

Sólidos en caldo, absorción de agua del grano y peso de testa, cotiledón y embrión

Los sólidos se cuantificaron llevando a resequedad $10 \mathrm{ml}$ de caldo de frijol cocido y la absorción de agua se midió dejando las semillas en remojo en agua purificada $18 \mathrm{~h}$, para proceder a la cocción. Estas determinaciones también se hicieron por triplicado. Para obtener el peso seco de las partes de las semillas, se procedió a remojar estas durante tres horas. Posteriormente, se tomaron cuatro muestras de cinco semillas cada una, y se separó manualmente la testa, los cotiledones y el embrión. Por último, se secaron al horno y se obtuvo el peso seco, llevando a peso constante (Pérez-Herrera et al., 2002; Iniestra-González et al., 2005).

\section{Análisis de elementos minerales}

La cuantificación de minerales se llevó a cabo usando un microscopio electrónico de barrido (JEOL JMS-6480LV) acoplado a una sonda dispersiva de rayos X (INCAx-sight Oxford Instruments) en pastillas de las harinas obtenidas de una prensa hidráulica CARVER (Liu et al., 2007; Raya-Pérez y AguirreMancilla, 2009). Se realizaron tres análisis por muestra.
Para cuantificar el hierro, se pesó $0,1 \mathrm{~g}$ de harina, del cual se realizó la extracción de hierro, utilizando $400 \mu 1$ de ácido nítrico al 3\% (v/v) durante 72 horas a $45^{\circ} \mathrm{C}$, luego se centrifugó y se recuperó el sobrenadante para la cuantificación espectrofotométrica a $535 \mathrm{~nm}$, usando BPS (ácido bathophenantrolin-disulfonico, Sigma-aldrich ${ }^{\mathrm{TM}}$ ) (Tamarit et al., 2006).

\section{RESULTADOS Y DISCUSIÓN}

Con el método utilizado para la extracción de las fracciones proteicas se obtuvo un contenido de proteína soluble total de $16,47 \%$, en el caso del Bayo Berrendo, y 14,53\% para el Patzcuareño. Del total de la proteína extraída, la fracción albúmina constituyó el 53,49\% para Bayo Berrendo, la de globulina representó el 19,80\%, la prolamina el 5,88\% y la glutelina el 20,8\%. Para Patzcuareño, la fracción albúmina constituyó el 30,90\%, globulina el 55,74\%, prolamina el $6,19 \%$ y glutelina el 7,08\%. Tomando en consideración que las proteínas solubles representan más del $50 \%$ de la proteína total, estas variedades muestran un buen contenido de la misma. En Ebenus ecretica, una leguminosa, la principal fracción proteica es de las glutelinas y la menor es la correspondiente a prolaminas (Syros et al., 2003). Guzmán- Maldonado et al. (2000) reportaron un contenido de $33 \%$ a $22 \%$ de proteína total y Pérez-Herrera et al. (2002) 23,5\% proteína total en promedio para frijol mexicano; por su parte, Serrano y Goñi (2004) dan un valor de 21,8\% de proteína total en frijol común negro; Montoya et al. (2008) encontraron un $24 \%$ proteína total. En cuanto a las faseolinas, en el Bayo Berrendo representan un 7\% y en el Patzcuareño un 6\%; aproximadamente la mitad del total de las proteínas solubles, valores acordes a lo encontrado por otros autores (Montoya et al., 2008).

El patrón electroforético para proteína total resultó muy semejante entre ambas variedades (Figuras 1 y 2 , carril 6 , respectivamente), destacándose bandas de alrededor de 95, 55 y $37 \mathrm{kDa}$. Se decidió correr geles con proteína total, proteínas de reserva y faseolinas para comparar entre ellas y establecer la más útil para la caracterización, dado que los diferentes autores utilizaron distintas fracciones proteicas sin discriminación aparente; por ejemplo, Syros et al. (2003) fraccionaron las proteínas; Mirali et al. (2007) usaron un extracto de proteína total y Sammour et al. 


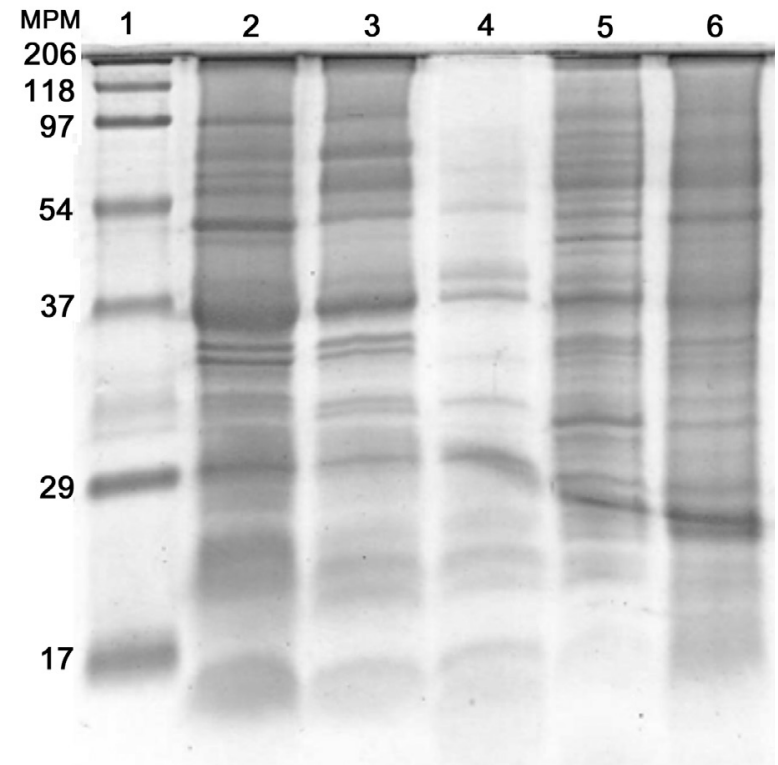

Figura 1. Perfil electroforético del frijol Bayo Berrendo. 1) MPM, Marcadores de peso molecular $(\mathrm{kDa}), 2$ ) Fracción albúmina, 3) Fracción globulina, 4) Fracción prolamina 5) Fracción glutelina, 6) Proteína total. Celaya, México. 2011.



Figura 2. Perfil electroforético de frijol Patzcuareño. 1) MPM, Marcadores de peso molecular (kDa), 2) Fracción albúmina, 3) Fracción globulina, 4) Fracción prolamina 5) Fracción glutelina, 6) Proteína total. Celaya, México. 2011.
(2007) usaron extracto de proteína cotiledonar total; Montoya et al. (2008) usaron faseolinas.

En la fracción albúmina (Figura 1, carril 2) casi a la altura del marcador de 54,8 kDa se observan dos bandas muy juntas e intensas en el Bayo Berrendo (flecha) y una sola en el Patzcuareño (Figura 2, carril 2), aunque, en contrapartida, en este se observan una banda muy visible entre los marcadores de 54,8 y 97,2 $\mathrm{kDa}$ que no se observa en el Bayo Berrendo.

La banda justo a la altura del marcador de 29,3 fue más intensa en el Bayo Berrendo y por debajo de este marcador se observan dos bandas intensas en el Patzcuareño y una sola en el Bayo Berrendo. En la fracción globulina no se detectaron diferencias entre ambos (Figura 1 y 2, carril 3, respectivamente). Ambas variedades lucen patrones electroforéticos muy semejantes en la fracción prolamina (Figura 1 y 2, carril 4); para la fracción glutelina (Figura 1 y 2, carril 5), se cuentan hasta 30 bandas; a la altura del marcador de 97,2 kDa se observa una banda más gruesa e intensa en la proteína de Bayo Berrendo y entre este marcador y el de $54,8 \mathrm{kDa}$ hay dos bandas finas pero intensas y una un poco más gruesa justo a la altura de este marcador de peso molecular.

En el Patzcuareño no se observaron bandas que resalten entre el marcador de 97,2 y el de 54,8 kDa. Otros autores reportan un número similar de bandas, pero para proteína total, lo que indica que el fraccionamiento de las distintas proteínas podría arrojar mejores resultados a fin de discriminar entre variedades (Sammour et al., 2007).

$\mathrm{El}$ amortiguador de boratos dio mejores resultados en cuanto a la definición del patrón electroforético que la extraída con $\mathrm{NaOH}$ (dato no mostrado). En cuanto a la proteína total, se observan algunas diferencias; entre los marcadores de 54,8 y $37,6 \mathrm{kDa}$, se aprecian tres bandas bien definidas en el Bayo Berrendo (flecha), pero con menor intensidad en Patzcuareño.

El desgrasado de harina antes de extraer las proteínas no fue indicado por Mirali et al. (2007). Syros et al. (2003) usaron harina desgrasada y sin desgrasar y no encontraron diferencias en el patrón electroforético de proteínas totales entre ambas harinas, lo que podría deberse a que usaron el sistema de electroforesis de Laemmli (1970), que es diferente al utilizado en este trabajo.

Para la extracción secuencial con base en solubilidad, la harina desgrasada con cloroformo-metanol fue la que dio bandas mejor definidas en los geles, mejor 


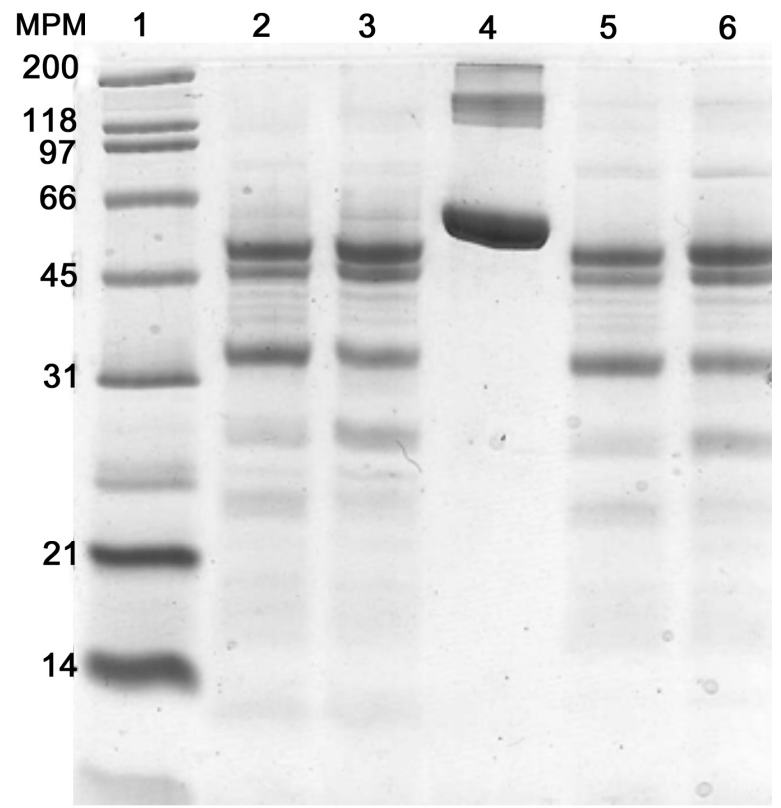

Figura 3. Perfil electroforético de las faseolinas. 1) MPM, Marcadores de peso molecular (kDa), 2) Faseolinas de frijol Bayo Berrendo, 3) Faseolinas de frijol Patzcuareño, 4) Albumina de suero bovino, 5) Faseolina de frijol Bayo Berrendo sin 2-mercaptoetanol, 6) Faseolina de frijol Patzcuareño sin 2-mercaptoetanol. Celaya, México. 2011.

aún que la harina desgrasada con hexanos (dato no mostrado). La harina sin desgrasar dio geles barridos sin bandas bien definidas (dato no mostrado). Syros et al. (2003) también reportan que la extracción de prolaminas con propanol no da bandas bien definidas, ni tampoco las extraídas con 2-cloroetanol y corridas en condiciones de electroforesis no desnaturalizante.
En las faseolinas de Patzcuareño (Figura 3, carril 3 ), se observan dos bandas finas por debajo del marcador de $66,2 \mathrm{kDa}$, dos bandas gruesas un poco por encima del marcador de $45 \mathrm{kDa}$. En las de Bayo Berrendo (Figura 3, carril 2), hay al menos dos bandas de proteína por debajo del marcador de $66,2 \mathrm{kDa}$, las dos gruesas a la altura del marcador de $45 \mathrm{kDa}$ y una muy delgada por debajo de las dos gruesas. Cuando no se añade 2-mercaptoetanol a las faseolinas, se observan dos bandas entre los marcadores de 66,2 y 97,4 kDa y entre los de 116,2 y $200 \mathrm{kDa}$.

Las proteínas pueden estar constituidas por dos a seis subunidades (Figura 3, carriles 5 y 6), con masas moleculares que van de los 54,4 a los 45,6 kDa (Montoya et al., 2008). Es importante estudiar con detalle las faseolinas pues las diferencias en estas, principalmente en cuanto a contenido de cisteína y metionina, influyen sobre la calidad de la proteína y su digestibilidad (Montoya et al., 2008), aunque en un estudio amplio entre variedades de frijoles mexicanas no se halló mucha variación en la digestibilidad (Pérez-Herrera et al., 2002). Para mejorar la digestibilidad se recomienda medir esta in vitro, tomando en cuenta además la composición aminoacídica de las faseolinas, a fin de obtener frijol con proteína de mejor calidad (Montoya et al., 2008).

En frijol se ha observado que a mayor capacidad de absorción de agua disminuye el tiempo de cocción, por lo que esta característica se evalúa durante la selección de variedades. Las variedades aquí estudiadas presentaron tiempos de cocción inferiores y capacidad de absorción de agua mayores a los valores reportados por Pérez Herrera et al. (2002). Esto permite recomendar estas variedades por estas características favorables, puesto que disminuye el gasto de tiempo y energía al cocinarlas (Cuadro 1).

Cuadro 1. Porcentaje de humedad, peso de 100 semillas, tiempo cocción y agua absorbida por dos variedades de frijol criollo. Celaya, México. 2011.

\begin{tabular}{lccccc}
\hline & Humedad $(\%)$ & $\begin{array}{c}\text { Peso de 100 } \\
\text { semillas }^{1}(\mathbf{g})\end{array}$ & $\begin{array}{c}\text { Tiempo cocción } \\
(\mathbf{m i n})\end{array}$ & $\begin{array}{c}\text { Agua absorbida } \\
\text { en 100 } \mathbf{g} \text { de } \\
\text { semilla }^{2}(\mathbf{g})\end{array}$ & $\begin{array}{c}\text { Actividad inhibi- } \\
\text { toria en fracción } \\
\text { faseolinas }\end{array}$ \\
\hline Bayo Berrendo & $10,11 \pm 0,15$ & $14,66 \pm 0,88$ & $64,30 \pm 3,78$ & $103,33 \pm 6,00$ & $355 \pm 139$ \\
Patzcuareño & $9,65 \pm 0,24$ & $32,61 \pm 1,85$ & $67,00 \pm 3,00$ & $87,50 \pm 4,35$ & $507 \pm 30$ \\
\hline
\end{tabular}

${ }^{1}$ La humedad y el peso de 100 semillas se determinó de semilla comercial.

${ }^{2}$ El tiempo de cocción y agua absorbida se determinó como se indica en Pérez-Herrera et al. (2002). 
Estas variedades mostraron un buen contenido mineral; Guzmán-Maldonado et al. (2000) reportaron de 84 a 280 ppm de Fe en frijol cultivado y silvestre, encontrando que este último tiene el doble de contenido que el cultivado, reportándose algunas colectas de frijol Rosa de Castilla, variedad nativa, con un contenido de este elemento de 65 ppm (Jiménez-Hernández et al., 2012); para el frijol negro también se reporta un valor de 68 ppm, aunque con baja biodisponibilidad (Serrano y Goñi, 2004), lo que podría explicar la variabilidad en las determinaciones pues la fracción extraíble difiere entre variedades. El Bayo contiene más fierro (18,8 ppm) que el Patzcuareño (3,63 ppm); si el tamaño de la semilla o el tipo de suelo influye en esta característica es algo que se sugiere explorar, como lo señalan Guzmán-Maldonado et al. (2000), sobre todo si hubiera un componente genético altamente influyente; Amirul Islam et al. (2006) reportan un contenido de Fe de 54,9 ppm.

El contenido de calcio para el Bayo Berrendo fue igual al promedio reportado para otras variedades y es prácticamente el mismo contenido que se obtuvo para el Patzcuareño (Figura 4), ambos dentro del intervalo reportado por otros autores, como Guzmán-Maldonado et al. (2000) quienes encontraron 7470 ppm de Ca pero Serrano y Goñi (2004) reportan 1310 ppm como contenido de $\mathrm{Ca}$ en frijol negro. Ambas variedades presentaron un alto contenido de potasio entre el $3 \mathrm{y}$ más del 5\% (Figura 4), Serrano y Goñi (2004) reportan un contenido de $\mathrm{K}$ de $2 \%$ para el frijol negro, más elevado que los reportados para otras variedades que

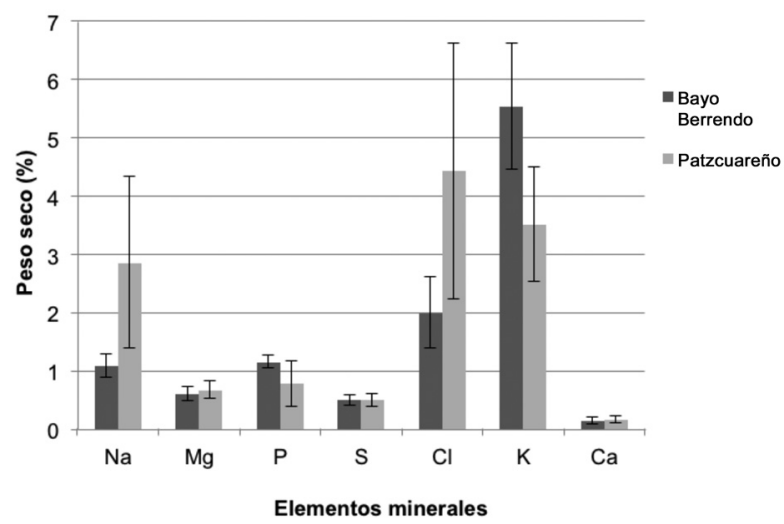

Figura 4. Composición mineral de las harinas enteras de frijol Bayo Berrendo y Patzcuareño. Celaya, México, 2011. es de 1,47\% (Amirul-Islam et al. 2006); esto podría estar influido por el tipo de suelo donde se desarrolla el cultivo. El análisis arrojó un contenido de magnesio muy semejante entre ambas variedades (Figura 4) más elevado que lo reportado para otras variedades lo cual es una característica sobresaliente; esto podría deberse al tipo de suelo en que son cultivadas; a este mineral se le está prestando mayor atención en relación a la salud de los consumidores; Serrano y Goñi (2004) reportan un contenido de $\mathrm{Mg}$ de 0,27\% para el frijol negro y Amirul-Islam et al. (2006) reportan un valor de $0,189 \%$. En el caso de Brassica, el contenido de calcio y magnesio tiene un gran componente hereditario (Broadley et al., 2008), lo mismo que el contenido de potasio. Existen relaciones recíprocas entre elementos; por ejemplo, la deficiencia de Fe eleva las concentraciones de Mn, Co, Zn, y Cd (Baxter, 2009). Se ha observado influencia genética para el contenido de $\mathrm{Zn}$ en grano pero también del tipo de suelo (JiménezHernández et al., 2012).

El contenido de azufre reportado por Amirul-Islam et al. (2006) es de 0,213\% y para el fósforo (P) es de 0,362\%; Serrano y Goñi (2004) reportan un contenido de P de 0,54\% para el frijol negro. Los valores de sodio encontrados son similares a los obtenidos por Serrano y Goñi (2004). Los datos aquí mostrados podrían podrían servir como guía sobre algunos parámetros a tomar en cuenta para posibles programas de mejoramiento.

Es conocida la presencia de inhibidores y otros factores antinutricionales en frijol y otras plantas; en este estudio se encontró actividad inhibitoria contra tripsina, principalmente en la fracción de faseolinas (Cuadro 1), acorde con otros reportes; Pérez-Herrera et al. (2002) obtuvieron una actividad inhibitoria de $0,75 \mathrm{mg} / \mathrm{g}$ a $1,9 \mathrm{mg} / \mathrm{g}$ ). Sin embargo, indican que en concentraciones pequeñas (o adecuadas) resultan benéficos para la salud (Serrano y Goñi, 2004). Así, fracciones como las faseolinas o albúminas podrían usarse a fin de purificar y caracterizar estos componentes y dilucidar su posible uso como alimento funcional o incluso terapéutico. La mayoría de los factores antinutricionales como los inhibidores de proteasas se inactivan con la cocción y quedan disponibles los aminoácidos que los constituyen, incluidos los azufrados, especialmente importantes en el caso del frijol desde el punto de vista nutricional (Pérez-Herrera et al., 2002; Serrano y Goñi 2004; Montoya et al., 2008).

Se extrajeron las proteínas de reserva de las dos variedades de frijol, con pequeñas diferencias en el 
patrón electroforético. Es deseable ahondar más en estas posibles diferencias, por ejemplo usando AFLPs o microsatélites. Usando una de estas técnicas moleculares (RAPD-ISSR), se encontró poca variabilidad entre variedades de frijol negro, incluyendo criollas y mejoradas (Vidal-Barahona et al., 2006), lo que se explica en parte por el hecho de que todas fueron de la misma región y, al parecer, las variedades criollas incluidas en el estudio se usaron para obtener las variedades mejoradas. Las diferencias en cuanto al contenido mineral, aunque también pequeñas, permiten la oportunidad de poder seleccionar esta característica.

Las variedades aquí estudiadas son de enredadera y con variabilidad en el color de la semilla. En cuanto al peso, estas resultan pequeñas en el caso del Bayo Berrendo (14,6 g/100 semillas); de acuerdo a los resultados reportados para frijol silvestre y asilvestrado, (entre 2 y 17,4 g/100 semillas), el Bayo Berrendo caería en esta categoría, valor muy cercano también del peso promedio ( $8 \pm 4 \mathrm{~g} / 100$ semillas) para frijol silvestre de la colección del CIAT (Guzmán-Maldonado et al., 2000; Montoya et al., 2008).

En la región de Colima, se tiene preferencia por un frijol de grano pequeño, al parecer debido a su sabor, aunque otros autores han encontrado que a menor tamaño de grano, mayor contenido de proteína, lo cual también lo haría más atractivo (Guzmán-Maldonado et al. 2000; Amirul-Islam et al., 2006; Montoya et al., 2008).

Las variedades de frijol domesticado del CIAT tienen un tamaño de semilla que va de $45 \pm 21 \mathrm{~g} / 100$ semillas, en tanto para el frijol común de México se reporta una media de 34,8 g/100 semillas, aunque para el Rosa de Castilla el peso reportado es de 40,2 g (Jiménez-Hernández et al., 2012).

Con respecto al frijol Patzcuareño se obtuvo un peso de 32,6 g/100 semillas que es prácticamente el doble que el del Bayo Berrendo. En este caso, parece que la preferencia de la gente se explica, en parte al menos, por ser un frijol criollo y cultivado de manera tradicional. El Bayo Berrendo y el Patzcuareño son granos bien cotizados. Este sobrepreso del Bayo Berrendo y Patzcuareño sin duda también los hace atractivos para que los productores sigan sembrándolos.

El contenido de humedad en las semillas es importante en términos de conservación y almacenamiento, valores encontrados en este estudio (Cuadro 1) son muy cercanos a los reportados por otros autores $(11,4$ a $12,9 \%)$ (Da Silva-Fialho et al., 2006).
La capacidad de absorción de agua fue del 100\% para el Bayo Berrendo y un poco menor para el Patzcuareño (90\%) y los tiempos de cocción son también muy semejantes; una buena capacidad de absorción de agua, mayor al 90\%, da tiempos cortos o no tan largos de cocción (menores a 110 minutos), aunque Serrano y Goñi (2004) reportaron tiempos de cocción desde 25 a 240 minutos, tomando en cuenta que algunas familias remojan el frijol toda la noche previa a la cocción; poco tiempo de cocción es una característica deseable desde el punto de vista de los consumidores (Cuadro 1) (PérezHerrera et al., 2002). La manera de cocer los frijoles es también importante. Hay variaciones en cuanto al tiempo empleado dependiendo de si se remojan o no los frijoles antes de cocerlos; esta práctica, aunque permite la salida de algunos nutrimentos, minerales principalmente, acorta el tiempo de cocción. El uso de olla de presión (express) es otra alternativa que tiene la particularidad de incrementar la fibra dietética soluble (Serrano y Goñi, 2004).

Otro parámetro importante para la aceptación de los consumidores es el espesor de caldo. El Bayo Berrendo presenta un mayor contenido de sólidos en caldo, aunque ambos presentan un buen contenido de sólidos, sin que llegue a deshacerse el grano durante la cocción (Cuadro 1). Pérez-Herrera et al. (2002) reportan un contenido de sólidos en caldo de $0,41 \%$. El caldo es importante por ejemplo, para dar de comer a los bebés y popularmente se le atribuyen cualidades nutricionales elevadas. El caldo puede contener hasta $2 \%$ de la proteína del frijol y $10 \%$ de los carbohidratos, además de otros componentes hidrosolubles, como vitaminas y minerales (Serrano y Goñi, 2004).

Dado que el Bayo Berrendo es de tamaño de grano pequeño y el Patzcuareño mediano, sería interesante explorar la variabilidad en esta característica, dado que durante la domesticación se ha seleccionado, en general, para tamaño grande de semilla. Se observó variación en el tamaño para el Patzcuareño, algunos lotes con semillas más grandes que otros, aunque conservando el aspecto general en forma y color. Esto podría deberse a diferencias en el manejo agronómico (Jiménez-Hernández et al., 2012). Dado que ambas variedades son de enredadera, se les podría usar en policultivos o en asociación con maíz. Se puede considerar la posibilidad de usar estas variedades en la búsqueda de materiales capaces de crecer y producir con escasos o nulos suministros en forma de fertilizantes químicos 
Cuadro 2. Comparación de medias mediante la prueba de Tukey, de tres variables de semilla en dos variedades de frijol. Celaya, México. 2011.

\begin{tabular}{lccc}
\hline Variedades & Peso embrión $(\mathbf{g})$ & Peso cotiledón $(\mathbf{g})$ & Peso testa $(\mathbf{g})$ \\
\hline Patzcuareño & $0,0214 \mathrm{a}^{*}$ & $1,36 \mathrm{a}$ & $0,1697 \mathrm{a}$ \\
Bayo Berrendo & $0,0117 \mathrm{~b}$ & $0,703 \mathrm{~b}$ & $0,0767 \mathrm{~b}$ \\
\hline
\end{tabular}

* Medias con la misma letra son estadísticamente iguales (Tukey, $\alpha=0,05$ ).

y/o con poca labor sobre el terreno, sobre todo en regiones similares a las que estén ya adaptados (Good et al., 2004; Jiménez Hernández et al., 2012).

En relación al peso de los componentes de la semilla de las variedades de frijol, la comparación de medias entre ambas variedades muestra diferencias altamente significativas, lo cual indica que son muy diferentes en cuanto a peso y tamaño de embrión y de cotiledones (Cuadro 2). No obstante lo anterior, son variedades muy aceptadas por los consumidores, característica importante desde el punto de vista comercial. El contenido de testa del Bayo Berrendo es de un 9,6\% y el del Patzcuareño de un 10,3\%; estos valores están dentro de los reportados por otros autores, aunque dentro de los valores relativamente altos, PérezHerrera et al. (2002) reportan un contenido promedio de testa para frijoles mexicanos de $8,2 \%$. El color de la testa además podría indicar una buena actividad antioxidante como ocurre para otras variedades (PérezHerrera et al., 2002; Iniestra-González et al., 2005). El contenido de cenizas resultó muy similar entre ambos materiales: el Bayo contiene 4,76\% y el Patzcuareño $5 \%$, similar a lo reportado por otros autores (OsorioDiaz et al., 2003).

Los carbohidratos solubles se resolvieron por cromatografía en placa fina (TLC), pero no se observaron diferencias entre ambas variedades. Se obtuvo una señal con un Rf de 1 correspondiente a glucosa, otra con $\mathrm{Rf}$ de 0,29 a sacarosa y dos más no identificadas con Rf de 0,13 y 0,04 . Aunque alguna de estas señales corresponde con una de las referencias, no se puede asegurar que se trate de fructanos, que fue lo que se usó como estándar de referencia. En el caso de la cuantificación de oligosacáridos, los datos mostraron contenidos similares para ambas variedades.

Las variedades son distinguibles entre sí morfológicamente, pero es importante realizar trabajo a nivel bioquímico y molecular para una mejor caracterización. Recientemente, el Bayo Berrendo se ha comenzado a comercializar de manera más amplia. Por otra parte, se espera que a pesar de la introducción de nuevas variedades mejoradas, el Patzcuareño sea conservado en algún grado in situ.

\section{AGRADECIMIENTOS}

Al Instituto Tecnológico Superior de Uruapan por las facilidades para utilizar el microscopio electrónico de barrido y a los ingenieros Martin Farfán y Rafael Tapia por el apoyo técnico. Al CONCyTEG convenios 09-11-K119-044 y 09-11-K662-095.

\section{LITERATURA CITADA}

Amirul-Islam, F.M., K.E. Basford, R.J. Redden, y S. Beebe. 2006. Preliminary evaluation of the common bean core collection at CIAT. Plant Genetic Research Newsletters 145:29-37.

Baxter, I. 2009. Ionomics: studying the social network of mineral nutrients. Current Opinion in Plant Biology 12:381-386.

Bradford, M.M. 1976. A rapid and sensitive method for the quantification of microgram quantities of protein utilizing the principle of protein dye binding. Analytical Biochemistry 72:248-252.

Broadley, M.R., J.P. Hammond, G.J. King, D. Astley, H.C. Bowen, M.C. Meacham, A. Mead, D.A.C. Pink, G.R. Teakle, R.M. Hayden, W.P. Spracklen, y P.J. White. 2008. Shoot calcium and magnesium concentrations differ between subtaxa, are highly heritable, and associate with potentially pleiotropic loci in Brassica oleraceae. Plant Physiology 146:1707-1720. 
Casañas, F., L. Bosch, M. Pujola, E. Sanchez, J. Sorribas, M. Baldi, y F. Nuez. 1999. Characterization of a common bean landrace (Phaseolus vulgaris L) of great culinary value and selection of a commercial inbred line. Journal of Science Food Agriculture 79:693-698.

Da Silva-Fialho, L., V. Monteze-Guimaraes, E. Gonçalvez de Barros, M. Alves-Moreira, L.A. Dos Santos-Dias, M.G. De Almeida-Oliveira, I. Chamel-Jose, y S. Tavares de Rezende. 2006. Biochemical composition and indigestible oligosaccharides in Phaseolus vulgaris L. seeds. Plant Food for Human Nutrition 61:87-89.

Erlanger, B.F., N. Kokowski, y W. Cohen. 1961. The preparation and properties of two new chromogenic substrates of trypsin. Archives of Biochemistry and Biophysics 95:271-278.

Gonçalves, N., J. Vioque, A. Clemente, R. Sánchez-Vioque, J. Bautista, y F. Millan. 1997. Obtención y caracterización de aislados proteicos de colza. Grasas y Aceites 48:282-289.

Good, A.G., A.K. Sharawat, y D.G. Muench. 2004. Can less yield more? Is reducing nutrient input into the environment compatible with maintanining crop production? Trends in Plant Science 9:597-605.

Guerrero-Zuñiga, L.A., y M.A. Rodríguez-Dorantes. 2009. Efecto de la presencia de fenantreno sobre la expresión de proteínas y la actividad enzimática radical de Cyperus hermaphroditus. Polibotánica 27:103-130.

Guzmán-Maldonado, H.S., J. Acosta-Gallegos, y O. Paredes-López. 2000. Protein and mineral content of a novel collection of wild and weedy common bean (Phaseolus vulgaris L.). Journal of the Science of Food and Agriculture 80:1874-1881.

Hall, M.B., W.H. Hoover, J.P. Jennings, y T.K. Miller-Webster. 1999. A method for partition neutral detergent soluble carbohydrates. Journal of the Science of Food and Agriculture 79:2079-2086.

Iniestra-González, J.J., F.J. Ibarra-Pérez, J.A. Gallegos-Infante, N.E. Rocha-Guzmán, y R.F. González-Laredo. 2005. Factores antinutricios y actividad antioxidante en variedades mejoradas de frijol común (Phaseolus vulgaris). Agrociencia 39:603-610.

Jiménez-Hernández, Y., J.A. Acosta-Gallegos, B.M. SánchezGarcía, y M.A. Martínez-Gamiño. 2012. Características agronómicas y contenido de Fe y $\mathrm{Zn}$ en el grano de frijol tipo Rosa de Castilla (Phaseolus vulgaris L.). Revista Mexicana Ciencias Agrícolas 3:311-325.

Laemmli, U.K. 1970. Cleavage of structural proteins during the assembly of the head of Bacteriophague T4. Nature 227:680-685.
Liu, D., I. Kottke, y D. Adam. 2007. Localization of cadmium in the root cells of Allium cepa by energy dispersive Xray analysis. Biologia Plantarum 51:363-366.

Mirali, N., S. El-Khouri, y F. Rizq. 2007. Genetic diversity and relationships in some Vicia species as determined by SDS-PAGE of seed proteins. Biologia Plantarum 51:660-666.

Montoya, C.A., P. Leterme, N.F. Victoria, O. Toro, W.B. Souffrant, S. Beebe, y J.P. Lailles. 2008. Susceptibility of phaseolin to in vitro proteolysis is highly variable across common bean varieties (Phaseolus vulgaris). Journal of Agricultural and Food Chemistry 56:2183-2191.

Nierth, A., y A. Jäschke. 2011. Radioactive phosphorylation of alcohols to monitor biocatalytic diels-alder reactions. PLoS ONE 6(6): e21391. doi:10.1371/journal. pone.0021391

Omae, H., A. Kumar, y M. Shono. 2012. Adaptation to high temperature and water deficit in the common bean (Phaseolus vulgaris L.) during the reproductive period. Journal of Botany doi:10.1155/2012/803413

Onder, M., y M. Babaoglu. 2001. Interactions amongst grain variables in various dwarf dry beans (Phaseolus vulgaris L.) cultivars. Journal of Agronomy \& Crop Science 187:19-23.

Osorio-Diaz, P., G. Méndez-Montealvo, E. Agama-Acevedo, J.J. Islas-Hernández, J. Sánchez-Muñoz, L.A. BelloPérez. 2003. Biodisponibilidad de almidón en dos variedades de frijol (Phaseolus vulgaris L.) en frijoles industrializados. Agrociencia 37:565-573.

Perazzini, R., D. Leonardi, S. Ruggeri, D. Alesiani, G. D’Arcangelo, y A. Canini. 2008. Characterization of Phaseolus vulgaris L. landraces cultivated in central Italy. Plant Food for Human Nutrition 63:211-218.

Pérez-Herrera, P., G. Esquivel-Esquivel, R. Rosales-Serna, y A. Acosta-Gallegos. 2002. Caracterización física, culinaria y nutricional de frijol del altiplano subhúmedo de México. Archivos Latinoamericanos de Nutrición 52:172-180.

Raya-Pérez, J.C., y C.L. Aguirre-Mancilla. 2009. Composición elemental de algunas plantas silvestres mexicanas. Revista Chapingo serie Ciencias Forestales y del Ambiente 15:95-99.

Romano, S.A., I. Tevés, y L. Cazón. 2010. Behaviour of mechanically y damaged seeds on the development and yield of normal and abnormal bean (Phaseolus vulgaris L.) Plants. IDESIA (Chile) 28(2):1-31.

Rubio, B. 2006. La política rural de Vicente Fox: entre la simulación y el desdén (2000-2006). Mundo Siglo XXI 5:41-51

AGRONOMÍA MESOAMERICANA 25(1):01-11. 2014 
Sammour, R.H., S.A. Radwan, y A. El-Koly. 2007. Genetic diversity in Phaseolus spp. as revealed by SDS-PAGE markers. Plant Genetic Resources Newsletters 151:6975.

Serrano, J., y I. Goñi. 2004. Papel del frijol negro Phaseolus vulgaris en el estado nutricional de la población guatemalteca. Archivos Latinoamericanos de Nutrición 54:36-44.

Schägger, H., y G. von Jagow. 1987. Tricine-sodium dodecyl sulfate polyacrylamide gel electrophoresis for the separation of protein in the range from 1-100 $\mathrm{kDa}$. Analytical Biochemistry 166:368-379.

Syros, T., T. Yupsanis, y A. Economou. 2003. Fractionation and electrophoretic patterns of Ebenus cretica. A preliminary survey as a tool in taxonomy. Biologia Plantarum 46:435-443.

Tandang-Silvas, M.R.G., E.M. Tecson-Mendoza, B. Mikami, S. Utsumi, y N. Murayama, N. 2011. Molecular design of seed storage proteins for enhanced food physicochemical properties. Annual Review of Food Science and Technology 2:59-73.

Tamarit, J., V. Irazusta, A. Moreno-Cermeño, y J. Ros. 2006. Colorimetric assay for the quantitation of iron in yeast. Analytical Biochemistry 351:149-151.

Vargas-Vázques, M.L.P., J.S. Muruaga-Martínez, J.A. AcostaGallegos, R. Navarrete-Maya, P. Pérez-Herrera, G. Esquivel-Esquivel, M.B.G. Irizar-Garza, y J.M. Hernández-Casillas. 2006. Colección núcleo de Phaseolus vulgaris L. del INIFAP: catálogo de accesiones de la forma domesticada. Libro técnico No. 10. SAGARPA, INIFAP, CIRCE, Chapingo, Estado de México, México.

Vidal-Barahona, A., L.C. Lagunes-Espinoza, E. ValadezMoctezuma, y C.F. Ortiz-García. 2006. Variabilidad morfológica y molecular de cultivares criollos y mejorados de frijol común en Tabasco, México. Revista Fitotecnia Mexicana 29:273-281. 